\title{
Targeting neuronal homeostasis to prevent seizures.
}

Fred Mulroe ${ }^{1 \dagger}$, Wei-Hsiang $\operatorname{Lin}^{1 \dagger}$, Connie Mackenzie-Gray $\operatorname{Scott}^{2}$, Najat Aourz ${ }^{3}$, Yuen Ngan Fan ${ }^{1, \infty}$, Graham Coutts $^{1,5}$, R Ryley Parrish ${ }^{2,}$, Ilse Smolders ${ }^{3}$, Andrew Trevelyan ${ }^{2}$, Robert Wykes ${ }^{4}$, Stuart Allan $^{1,5}$, Sally Freeman ${ }^{4}$, Richard A. Baines ${ }^{1 *}$.

${ }^{\dagger}$ Authors contributed equally

${ }^{1}$ Division of Neuroscience and Experimental Psychology, School of Biological Sciences, Faculty of Biology, Medicine and Health, University of Manchester, Manchester Academic Health Science Centre, Manchester, UK.

${ }^{2}$ Institute of Neuroscience, University of Newcastle, UK.

${ }^{3}$ Department of Pharmaceutical Chemistry, Drug Analysis and Drug Information, Research group Experimental Pharmacology, Center for Neurosciences, Vrije Universiteit Brussel, Brussels, Belgium.

${ }^{4}$ Division of Pharmacy \& Optometry, School of Health Sciences, Faculty of Biology, Medicine and Health, University of Manchester, Manchester Academic Health Science Centre, Manchester, UK.

${ }^{5}$ Geoffrey Jefferson Brain Research Centre, The Manchester Academic Health Science Centre, Northern Care Alliance NHS Foundation Trust, University of Manchester, UK

Present address:

${ }^{\infty}$ Imagen, Enterprise House, Lloyd Street North, Manchester, M15 6SE, UK

\$Department of Cellular and Molecular Biology, Xenon Pharmaceuticals, Burnaby, BC, Canada

*corresponding author: Richard.Baines@manchester.ac.uk 
Manipulating neuronal homeostasis, which enables neurons to regulate their intrinsic excitability, offers an attractive opportunity to prevent seizures. However, no anticonvulsant compounds have yet been reported that directly manipulate neuronal homeostasis. Here, we describe a novel class of anticonvulsant compounds, based on 4-tert-butyl-benzaldehyde (4-TBB), with a mode-of-action that includes increased expression of the homeostatic regulator Pumilio (Pum). In Drosophila and mouse we use a pentylenetetrazole (PTZ) induced seizure model, and an electrically induced seizure model for refractory seizures to evaluate anticonvulsant efficacy. The pyrazole analogue (RAB216) demonstrates best efficacy, protecting $50 \%$ of mice from PTZ-induced seizure. Knock-down of Pum, in Drosophila, blocks anticonvulsive effects, whilst analysis of validated Pum targets show significant reductions following exposure of mouse brain to 4-TBB. This study provides proof-ofprinciple that anticonvulsant effects can be achieved through regulation of neuronal homeostasis and identifies a chemical lead compound for future development.

KEY WORDS: Drosophila, Seizure, Neuronal homeostasis, Mouse, Pumilio, Translational regulation 


\section{Introduction}

Neuronal homeostasis provides an attractive target to achieve therapeutic control of epilepsy. This is because homeostatic mechanisms ostensibly oppose extremes of neuronal activity that are associated with seizures. By maintaining neuronal activity patterns at physiologically relevant 'setpoints', neuronal homeostasis acts to ensure stability of both neuron and network function across the life-course ${ }^{1}$. However, to date, neuronal homeostasis has not been specifically targeted for clinical benefit.

Pumilio (Pum) homeostatically maintains action potential firing rates within a set-range ${ }^{2}$. A translational repressor, Pum binds mRNA transcripts and reduces de novo protein synthesis, with increased Pum expression occurring in neurons exposed to increased synaptic excitation.

Conversely, as synaptic excitations fall, Pum expression is reduced ${ }^{3}$. The 3 '-UTR of Pum-regulated transcripts usually contain one or more copies of a Pum-Response Element (PRE: UGUANAUA, where $\mathrm{N}$ is $\mathrm{A}, \mathrm{C}, \mathrm{G}$ or $\mathrm{U})^{4}$. Analysis of both Drosophila and mammalian transcriptomes identifies more than 1000 transcripts that contain one or more PREs, consistent with a broad regulatory role ${ }^{5}$. However, Pum requires additional co-factors (including Nanos and Brain-tumor) and the actual effect of Pum is likely dictated by both the number and proximity of these additional binding elements, in addition to the number of PREs ${ }^{6,7}$. In mammals, regulated transcripts that have potential to influence neuron activity include $\mathrm{Na}_{\mathrm{v}} 1.6(\mathrm{SCN} 8 \mathrm{~A})^{2}$ and GLUR2 (AMPA receptor) ${ }^{8}$. Pum-dependent homeostatic translational repression of $\mathrm{Na}_{\mathrm{v}} 1.6$, in rat cortical pyramidal neurons, reduces the amplitude of expressed voltage-gated $\mathrm{Na}^{+}$current $\left(\mathrm{I}_{\mathrm{Na}}\right)$ and lowers action potential firing frequency ${ }^{2}$. Down-regulation of AMPA receptor expression may also be expected to be antiepileptic, evidenced by the anti-epileptic compound, perampanel, which is an allosteric antagonist of AMPA receptors ${ }^{9}$. 
Whilst Drosophila has one pum gene, mammals express two highly similar variants (pum 1 \& 2) that are co-expressed, and which bind identical RNA motifs and, thus, appear to act redundantly ${ }^{10-}$ 12. Seizure occurrence could reflect reduced homeostatic capability and it is significant that recent studies suggest reduced Pum contributes to epilepsy. Specifically, i) puml or 2 haploinsufficiency is associated with spontaneous seizures in mice ${ }^{13-15}$, ii) Pum2 expression is reduced in human patients suffering temporal lobe epilepsy and in rat hippocampus following pilocarpine-induced seizure ${ }^{16}$, and iii) dpum expression is reduced in Drosophila genetic seizure mutations ${ }^{17}$. In the latter, transgenic up-regulation of dPum is potently anticonvulsant in these same Drosophila mutations ${ }^{17}$.

Based on a screen to identify chemicals that increase expression and/or stability of dPum, we identified avobenzone, which secondary screens show is anticonvulsant in seizure-sensitive Drosophila ${ }^{17}$. However, the physiochemical properties of avobenzone are not compatible with clinical use. Thus, in this study, we report the identification of an avobenzone analogue, 4-tertbutyl-benzaldehyde (4-TBB), that is anticonvulsant and has properties more consistent with clinically active compounds. We show that 4-TBB and analogues (specifically the pyrazole RAB216) are active against a range of Drosophila seizure mutants and, significantly, reduce severity of both pentylenetetrazol (PTZ)- and pharmacoresistant electrically $(6 \mathrm{~Hz})$-induced seizures in mouse. Reduction of seizures, in fly and mouse, is accompanied by increased expression of Pum. We further report down-regulation of known Pum targets following exposure of mouse brain to 4TBB thus validating proposed mode-of-action.

\section{Results}

\section{4-TBB suppresses seizure behaviour in Drosophila}

Single Drosophila gene mutations increase seizure-like activity in response to electrical shock ${ }^{18}$. In a prior study, we identified avobenzone to both increase dpum expression and reduce seizure severity in Drosophila ${ }^{17}$. Avobenzone is poorly soluble and therefore we identified a breakdown 
product, 4-TBB to be a better candidate for analysis of mode-of-action. We tested the anticonvulsant activity of 4-TBB in three diverse seizure mutants to demonstrate wide applicability. Exposure to drug (1.2 mM contained within food) was sufficient to reduce electroshock-induced seizure duration in bangsenseless $^{1}$ ( para $^{\text {bss }}$; $\mathrm{Na}_{\mathrm{v}}$ hypermorph, $194 \pm 116$ vs. $348 \pm 101 \mathrm{~s}, 4-\mathrm{TBB}$ vs. control, $\mathrm{n}=30, p<0.001$ ), easilyshocked (eas; ethanolamine kinase deficiency, $160 \pm 81$ vs. $232 \pm$ $124 \mathrm{~s}, \mathrm{n}=30, p=0.011)$ and julius seizure (jus; a transmembrane domain protein of undetermined function, $197 \pm 124$ vs. $266 \pm 112 \mathrm{~s}, \mathrm{n}=30, p=0.03$, Fig. 1A). The level of seizure suppression observed for 4-TBB, in all mutations, is favourable compared to phenytoin (PHE, c.f. Fig. 1D).

The expression of $d p u m$ is reduced in para $^{b s s}$ mutants and, moreover, increasing dpum expression in this mutant background is sufficient to suppress seizure activity in response to electroshock ${ }^{17}$. To determine whether the anticonvulsant effect of 4-TBB is associated with up-regulation of $d p u m$, we used a dpum-minimal promoter construct upstream of GAL4 (dpum-GAL4) to drive expression of UAS-luciferase (UAS-luc) ${ }^{19}$. Exposure of $d p u m$-GAL4>UAS-Luc flies to 4-TBB (1.2 mM in food) resulted in a significant increase in luciferase activity $(2.7 \pm 1.5$ fold change, $\mathrm{n}=5, p=0.03$, vehicle control set as 1) (Fig. 1B). We adopted this approach because available anti-Pum antibodies (designed to rodent Pum1 and Pum2) do not work well in Drosophila. We also observed a significant increase in dpum transcript abundance, measured by QRT-PCR, of $\sim 60 \%(1.6 \pm 0.3$ fold change, $\mathrm{n}=5, p=0.002$, vehicle control set as 1) following exposure to 4-TBB (Fig. 1C). Finally, we find that the anticonvulsive activity of 4-TBB is significantly diminished when dpum expression is reduced in the CNS, via targeted expression of an RNAi construct (Fig. 1D). PHE, which has a different mode of action ${ }^{20}$, remains active under these conditions. We conclude that the mode-ofaction of 4-TBB requires the presence of dPum and increases expression of this homeostatic regulator. 


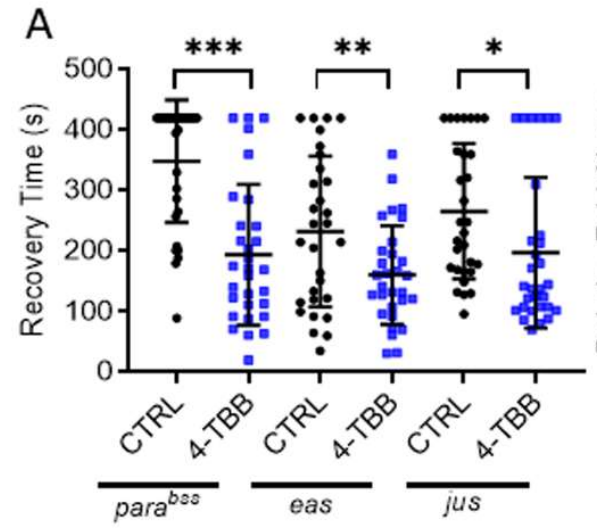

B
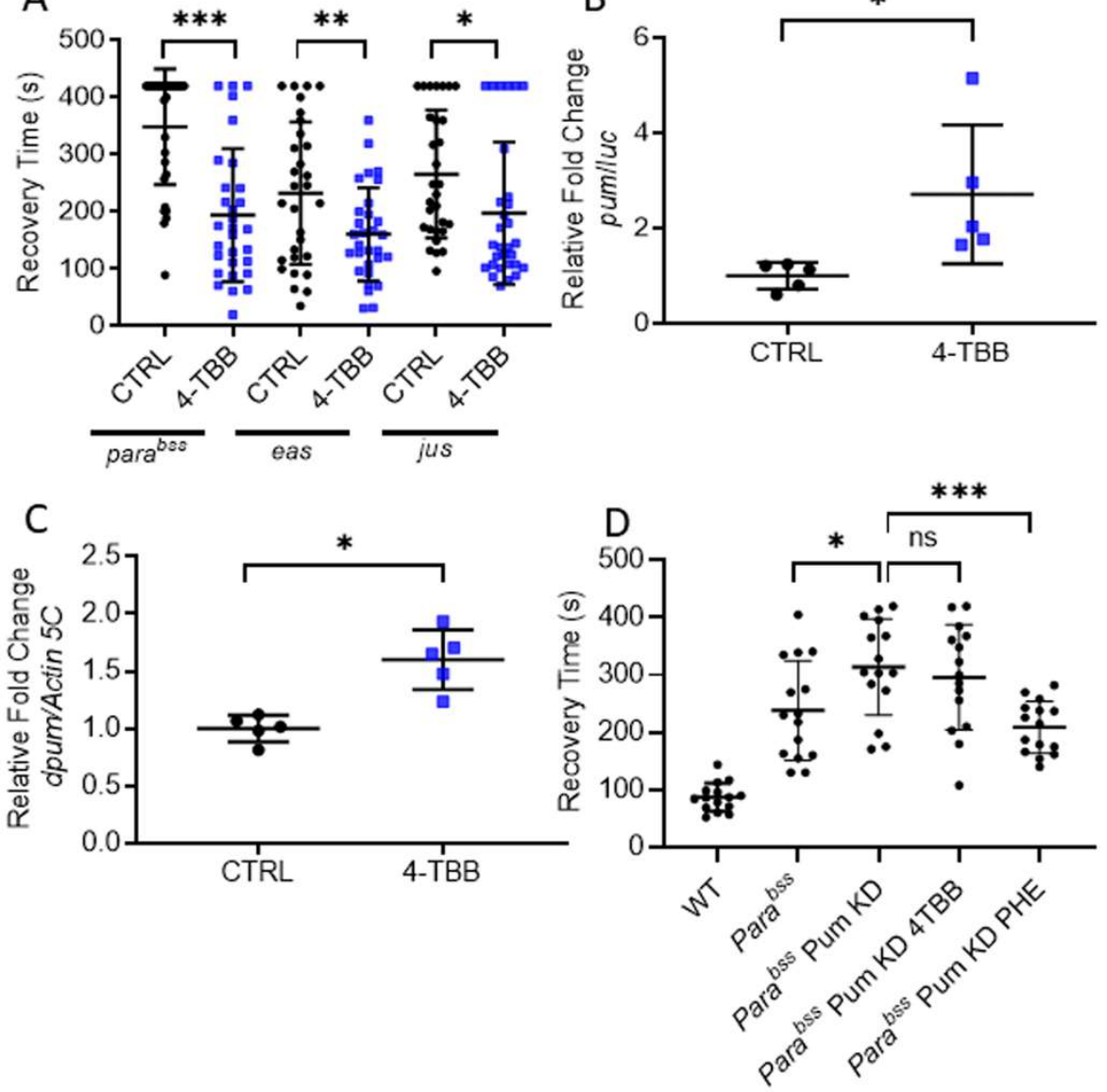

Figure 1. 4-TBB is anticonvulsant in Drosophila.

A) An anticonvulsant effect of 4-TBB is present in three independent Drosophila seizure mutations, para ${ }^{\text {bss }}$ $(p<0.001)$, eas $(p=0.011)$ and jus ( $p=0.03)$ (unpaired $t$-tests, $\mathrm{n}=30$ larvae for each treatment). B) Identical exposure of transgenic dpum promoter-

GAL4>UAS-Luciferase (Luc) larvae to 4-TBB results in significant upregulation of Luc expression $(p=0.03$, unpaired $t$-test, $\mathrm{n}=5$ independent samples / 10 CNS per sample). C) Ingestion of 4 -TBB is sufficient to increase $d p u m$ mRNA abundance measured by QRT-PCR ( $p$ $=0.002$, unpaired $t$-test, $\mathrm{n}=$

5 independent samples / 20 CNS per sample). D) RNAi-mediated knockdown of dpum expression in para $^{\text {sss }}$ expectedly increases seizure recovery time ${ }^{17}(p=0.03)$. Exposure to 4-TBB is ineffective in this background $(p=0.87)$, whilst exposure to phenytoin (PHE, $2 \mathrm{mM}$ in food) remains anticonvulsant $(p=0.002)$. Significance tested using a one-way ANOVA $\left(\mathrm{F}_{(4,70)}=24, p=0.0009\right)$ followed by Dunnett's post-hoc test (multiple experimental groups). Wildtype (WT) recovery time is shown for comparison. Bars report means $\pm \mathrm{SD}(\mathrm{n}=15) . *=p<0.05, * *=p<0.01, * * *=p<0.001, \mathrm{~ns}=$ not significant.

\section{4-TBB reduces epileptiform activity in mouse hippocampal culture slices}

Incubation of acutely-harvested mouse brain slice with 4-TBB (1.2mM) for $2 \mathrm{~h}$ was sufficient to produce a significant increase in Pum2 expression (Pum1 not measured), as determined by Western Blot (2.5 \pm 0.3 fold compared to vehicle-treated controls which were the corresponding slice taken from the opposite hemisphere incubated in vehicle only, set at $1, \mathrm{n}=5, p=0.002$, data not shown). Addition of 4-TBB (1.2mM) to mouse organotypic hippocampal slices, in which epileptiform 
bioRxiv preprint doi: https://doi.org/10.1101/2022.03.07.483229; this version posted March 7, 2022. The copyright holder for this preprint (which was not certified by peer review) is the author/funder, who has granted bioRxiv a license to display the preprint in perpetuity. It is made available under aCC-BY 4.0 International license.

activity was already established (Fig. 2A-B), led to a progressive reduction in epileptiform activity ( $p<0.0001 ; \mathrm{n}=7$ and 8, control vs. 4-TBB, respectively) compared to controls (vehicle added) that was apparent by $1 \mathrm{~h}$, and maximal after $3 \mathrm{~h}$ (Fig. 2C-D).

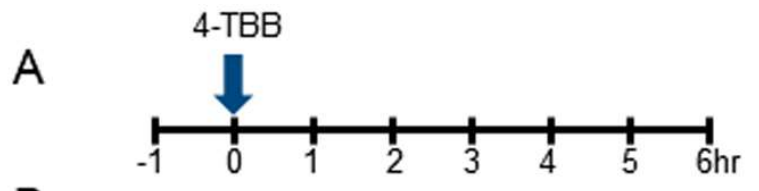

B
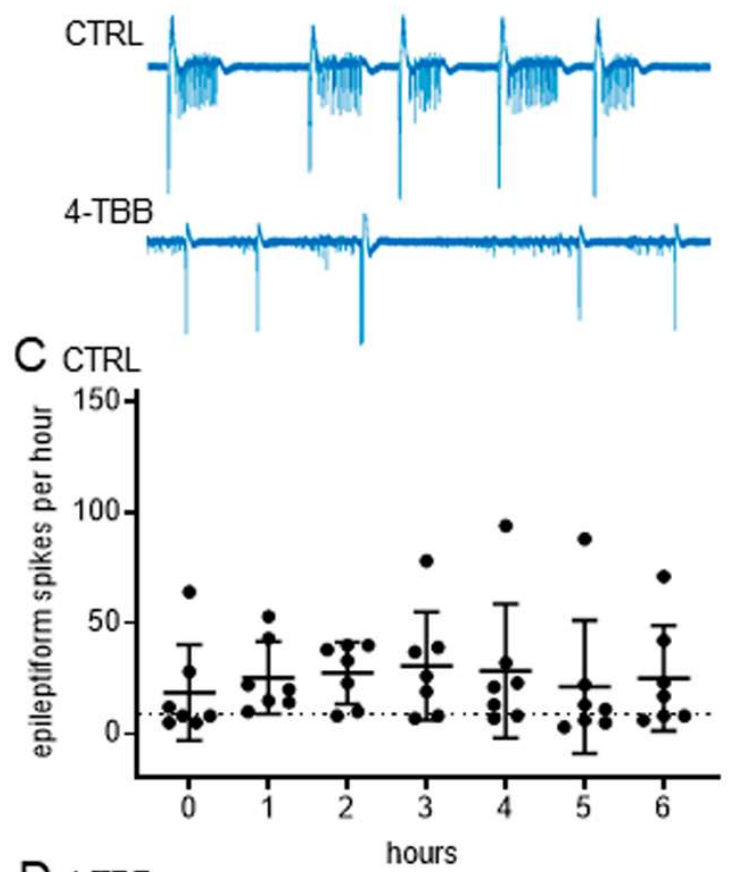

D 4-TBB

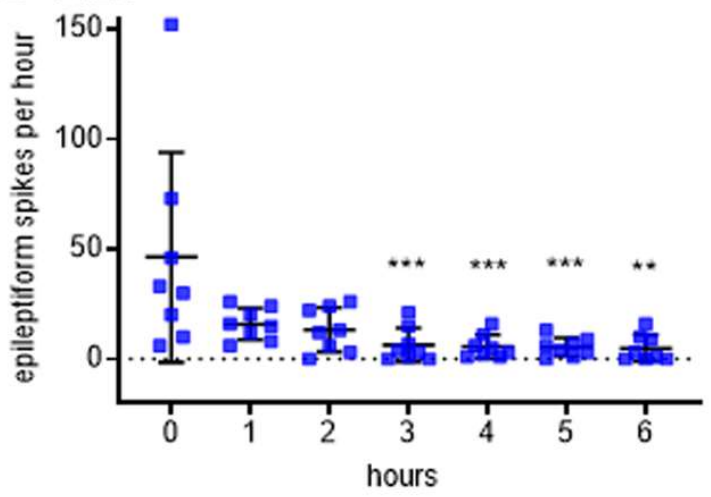

Figure 2. 4-TBB is anticonvulsant in mouse brain slice.

A) Hippocampal slices (7-14 DIV) that exhibited epileptiform activity were exposed to 4 -TBB at time ' 0 ' during a $7 \mathrm{hr}$ recording period. B) Representative extracellular recordings of local field potentials (LFPs) showing seizure activity in un-treated (CTRL) and treated (4-TBB) slices. C). Untreated slices (CTRL) show robust epileptiform activity throughout the recording period. D) 4TBB significantly reduces frequency of epileptiform activity, $\mathrm{n}=7$ (CTRL) and $\mathrm{n}=8$ (4TBB) independent slices, respectively. Significance for the effect of 4-TBB $v s$. CTRL was tested using a two-way ANOVA $\left(\mathrm{F}_{(13,84)}=5.823\right.$, $p<0.0001)$. Analysis of the effect of 4-TBB shows that the reduction in epileptiform activity is significant from $3 \mathrm{hrs}$ onwards $\left(\mathrm{F}_{(13,91)}=2.473, p\right.$ $=0.0063$ Š́́dák's multiple comparisons test). Bars show means $\pm \mathrm{SD} . * *=p<0.01, * * *=p<0.001$.

\section{4-TBB reduces induced PTZ-induced seizure in mice}

Mice were exposed to 4-TBB (800 mg/kg, s.c. injection) or saline vehicle (CTRL), once per day for 3 days. We observed no overt change in behaviour, nor weight loss, during the test-period. Four 
bioRxiv preprint doi: https://doi.org/10.1101/2022.03.07.483229; this version posted March 7, 2022. The copyright holder for this preprint (which was not certified by peer review) is the author/funder, who has granted bioRxiv a license to display the preprint in perpetuity. It is made available under aCC-BY 4.0 International license.

hours after the last injection on day 3, seizures were induced by PTZ (60 mg/kg, s.c. injection).

Latency to onset of Straub tailing (a first indicator of seizure) was significantly delayed in the 4-

TBB-exposed group (215.0 \pm 81.0 vs. $138.3 \pm 47.3 \mathrm{~s}, \mathrm{n}=12$ and 16 , respectively, $p=0.004$, Fig.

$3 \mathrm{~A})$. Time to onset of first generalised tonic-clonic seizure was also significantly delayed $(239 \pm 59$

vs. $166.8 \pm 44.1 \mathrm{~s}, 4$-TBB vs. saline control, $\mathrm{n}=12$ and 16 , respectively, $p=0.001$, Fig. $3 \mathrm{~B}$ ). To

confirm the expectation that this anticonvulsive effect of 4-TBB was associated with up-regulation

of Pum, post mortem brains were probed by Western blot. Expression of Pum1 and Pum2 were

significantly up-regulated in 4-TBB-exposed mice $(1.4 \pm 0.3$ and $1.2 \pm 0.2$ fold increase, $p<0.0001$ and $p=0.01$, respectively, Fig. 3C) compared to the saline controls (set as 1).

A Straub tail

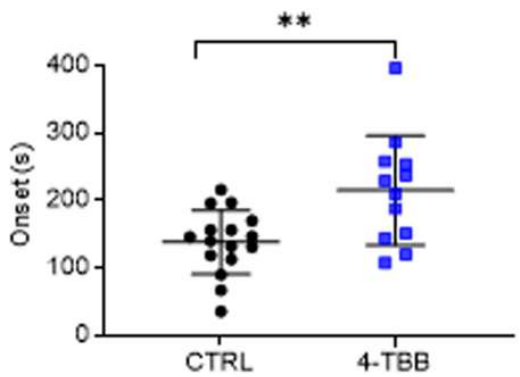

C
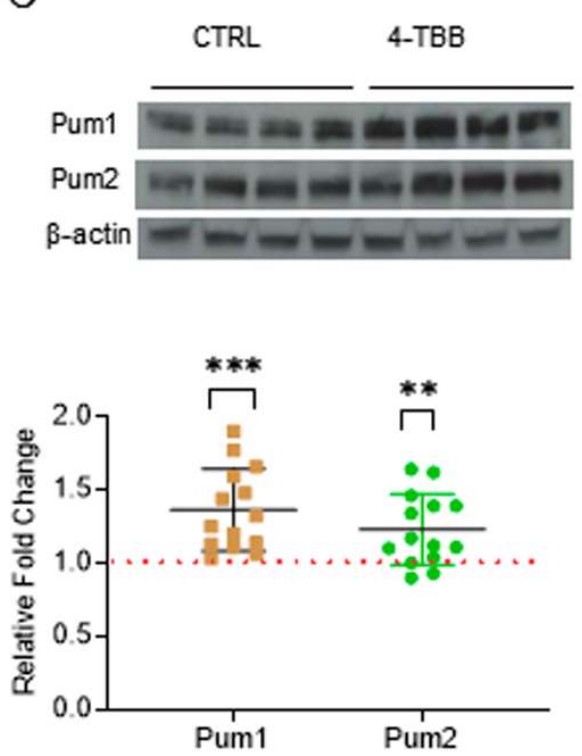

B Tonic-clonic seizure
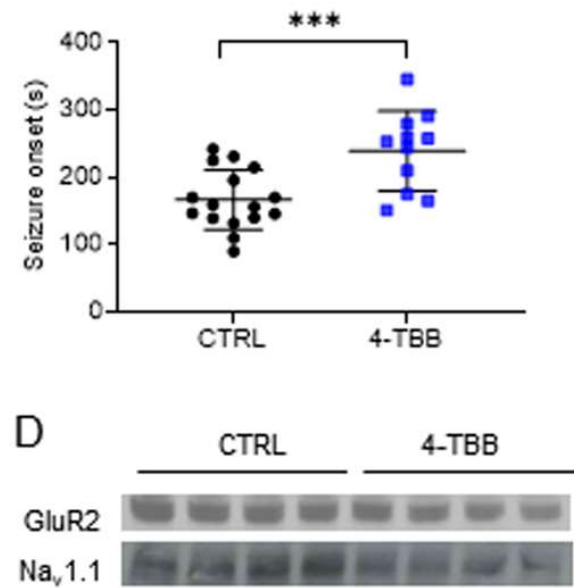

$\mathrm{Na}_{\mathrm{v}} 1.2$

$\mathrm{Na}_{\mathrm{y}} 1.6$

$\beta$-actin

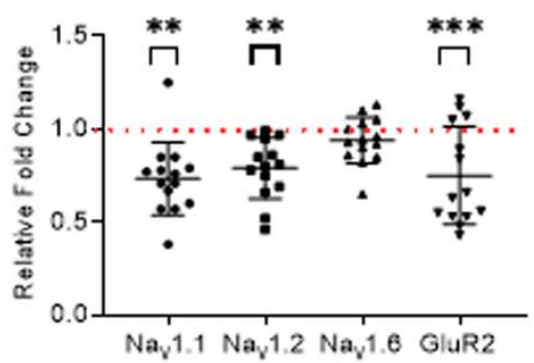

Figure 3. 4-TBB is anticonvulsant in the mouse PTZ-induced seizure model.

Exposure to 4-TBB increases time to onset of $\mathbf{A}$ ) Straub tail $(p=0.004)$ and B) generalised tonic-clonic seizure $(p=0.001)$. C). Post-mortem analysis of brains, taken from the mice used in the assay, shows expression of Pum1 $(p<$ $0.0001)$ and Pum2 ( $p=$ 0.01 ), is up-regulated in animals treated with 4-TBB compared to controls (set as $1)$. Bars show means \pm SD. Inset shows an example Western Blot. Significance was tested in A - C using unpaired $t$-tests. D) Western Blot analysis of $\mathrm{Na}_{\mathrm{v}} 1.1, \quad 1.2, \quad 1.6$ and GLUR2 expression in mouse brain show that exposure to 4-TBB is sufficient to down-regulate $\mathrm{Na}_{\mathrm{v}} 1.1(p=0.005), 1.2(p=0.007)$, and GLUR2 ( $p$ $=0.001)$, but not $\mathrm{Na}_{\mathrm{v}} 1.6(p=0.75) . \mathrm{n}=14$, Bars show means \pm SD. Significance was tested using 
ANOVA $\left(\mathrm{F}_{(4,65)}=6.7, p=0.0001\right)$ followed by Dunnett's post-hoc test (multiple experimental groups). Inset shows an example Western Blot. $* *=p<0.01, * * *=p<0.001$

Validated Pum-dependent regulated transcripts, in mammals, include $N a_{v} 1.1$ (SCN1A), $N a_{v} 1.6$ (SCN8A) and GLUR2 (aka GLUR-A, AMPA receptor) ${ }^{2,8,21}$. Bioinformatic analysis of expressed mRNAs also identifies a putative PRE motif in $N a_{V} 1.2$ (SCN2A), indicative that this channel variant is also regulated by Pum ${ }^{12}$. Western blot (Fig. 3D) of the same brain extracts, as above, shows that the expression level of Nav1.1, $\mathrm{Na}_{\mathrm{v}} 1.2(0.73 \pm 0.2$ and $0.79 \pm 0.16, p=0.005$ and 0.007 , respectively, $\mathrm{n}=14)$ and GLUR2 $(0.75 \pm 0.26, p=0.001, \mathrm{n}=14)$ were significantly reduced in brain tissue exposed to 4-TBB. No change was observed for $\mathrm{Na}_{\mathrm{v}} 1.6(0.94 \pm 0.12, p=0.75)$.

\section{Identification of a more potent 4-TBB analogue}

The above data shows proof-of-principle that a compound that effects increased Pum activity has significant potential as an anticonvulsant. However, the active concentration of 4-TBB required for significant effect, in the in vivo mouse PTZ-induced seizure assay $(800 \mathrm{mg} / \mathrm{kg})$ is relatively high compared to other clinically used CNS compounds. Testing $4-\mathrm{TBB}$ at a lower dose $(400 \mathrm{mg} / \mathrm{kg}) \mathrm{did}$ not result in statistically significant effects on seizure (data not shown). To identify a more potent analogue, we exploited Drosophila para $^{\text {bss }}$ to screen for anticonvulsive activity of a diverse set of fourteen (synthesised or purchased) 4-TBB analogues (Fig. 4, all structures and chemical properties are shown in sTable 1). We identified 4-(3,5-dimethyl-1H-pyrazol-4-yl)benzoic acid (hereafter termed RAB216) to be potently active against para $^{\text {bss }}$ at both $2 \mathrm{mM}$ (concentration added to food, Fig. 4A) and at 0.1mM (4-TBB was inactive at this level, data not shown). Analysis of mouse brain exposed to RAB216 (200mg/kg, once per day for 3 days), showed a significant increase in Pum2 expression $(p=0.01)$, and a smaller (but not significant, $p=0.07$ ) increase in Pum1 (Fig. 5A). We 
bioRxiv preprint doi: https//doi.org/10.1101/2022.03.07.483229; this version posted March 7, 2022. The copyright holder for this preprint (which was not certified by peer review) is the author/funder, who has granted bioRxiv a license to display the preprint in perpetuity. It is made available under aCC-BY 4.0 International license.

also screened for effect of valproate (VPA, an effective anticonvulsant in the PTZ-induced seizure assay) and saw no change to either Pum1 or 2 (Fig. 5B). Consistent with its heightened potency to increase Pum2 (c.f. Fig. 3C), RAB216 was $>4 \mathrm{x}$ more active than 4 -TBB in protecting mice against PTZ-induced seizures, being significantly active at 200mg/kg (Fig. 5C-D), a dose which prevented the induction of seizure in $50 \%$ of animals tested $(p=0.05$, Fig. $5 \mathrm{E})$. By contrast, a repeat of $4-\mathrm{TBB}$ exposure $(800 \mathrm{mg} / \mathrm{kg})$, in this assay, prevented tonic-clonic seizures in only $30 \%$ of animals during the 20min observation period (not significantly different to CTRL, data not shown). This provides confidence that further, yet more active chemical structures, may be discovered.

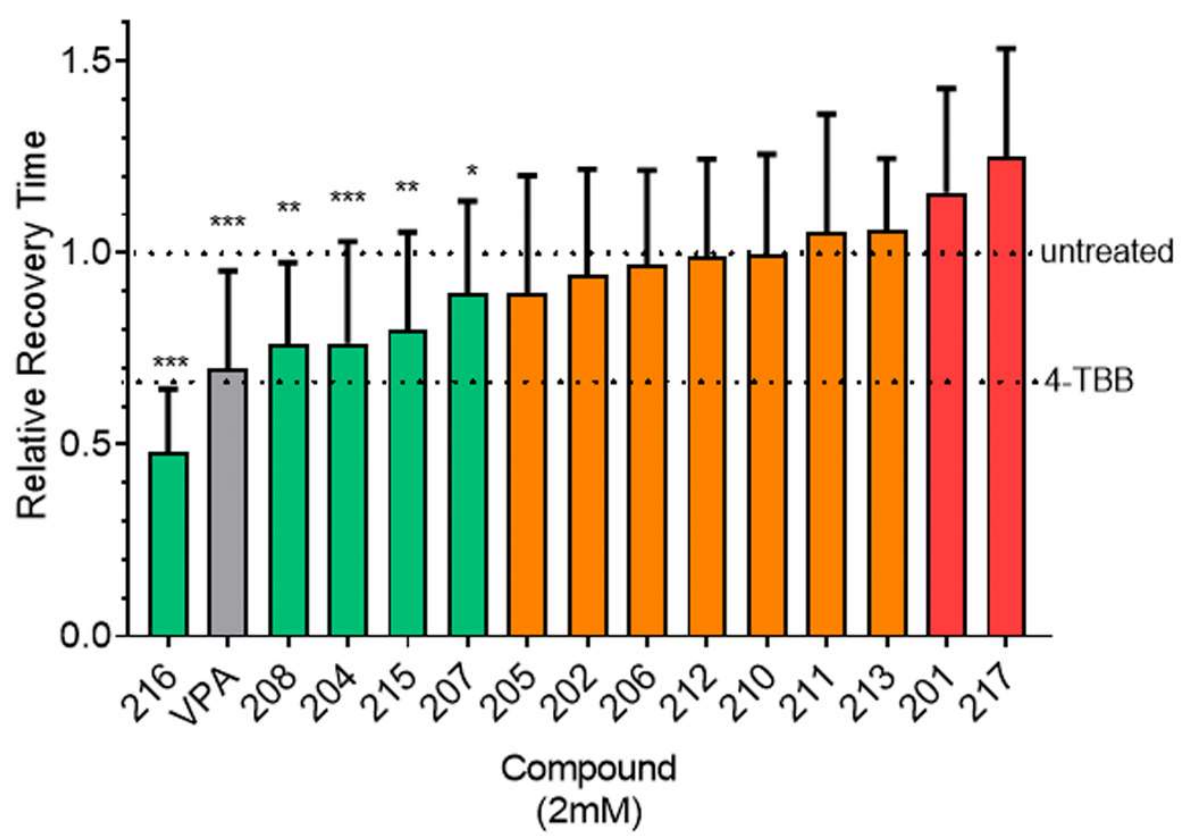

Figure 4. Identification of a more active 4-TBB analogue.

4-TBB analogues (structures shown in sTable 1) identified a number of active compounds, of which 4-(3,5-dimethyl-1H-pyrazol-4-yl) benzoic acid (RAB216) was the most potent. Relative recovery time (recovery time normalised to $\mathrm{para}^{b s s}$ run at the same time as drugs) was calculated as a ratio of the treatment group ( para $^{\text {bss }}+$ compound) recovery time compared to the corresponding untreated group ( para $^{b s s}$ - compound) from that week of screening. Green denotes a significant reduction in recovery time, orange denotes no change and red a proconvulsant effect. Sodium valproate (VPA, gray bar) was included as an additional control. $*=p<0.05, * *=p<0.01, * * *=p<0.001$. (unpaired $t$-tests, between para $^{\text {bss }}$ - compound vs. para ${ }^{\text {bss }}+$ compound). 
bioRxiv preprint doi: https://doi.org/10.1101/2022 03.07.483229; this version posted March 7, 2022. The copyright holder for this preprint (which was not certified by peer review) is the author/funder, who has granted bioRxiv a license to display the preprint in perpetuity. It is made available under aCC-BY 4.0 International license.

Finally, we tested RAB2 16 using a $6 \mathrm{~Hz}$ electrically-induced seizure assay, which has utility to model drug-refractory epilepsy ${ }^{22}$. Using the same drug administration protocol as for the PTZinduced seizure assay, prior exposure to RAB216 (200mg/kg) significantly reduced the length of induced seizure in this assay $(p=0.013, \mathrm{n}=9$, Fig. 5F). By contrast, 4-TBB $(800 \mathrm{mg} / \mathrm{kg})$ did not significantly reduce seizure duration $(p=0.1$, data not shown).

A

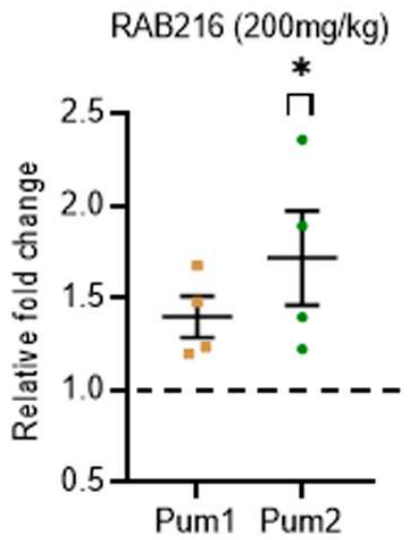

C

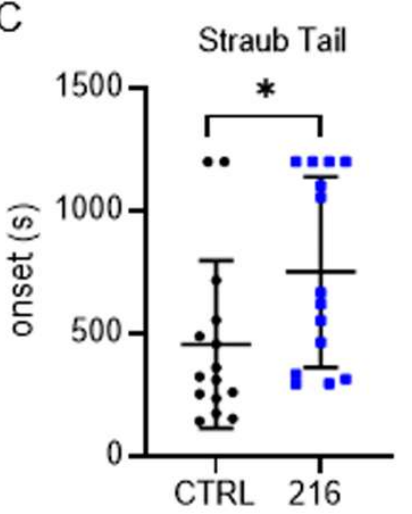

$\mathrm{E}$

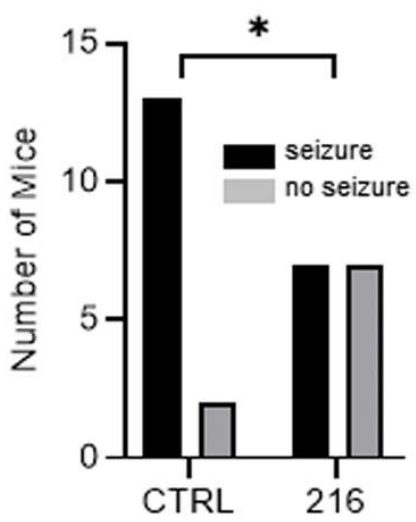

B $\quad$ VPA $(200 \mathrm{mg} / \mathrm{kg})$

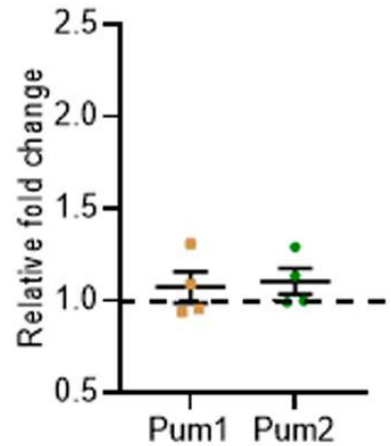

D Tonic-clonic
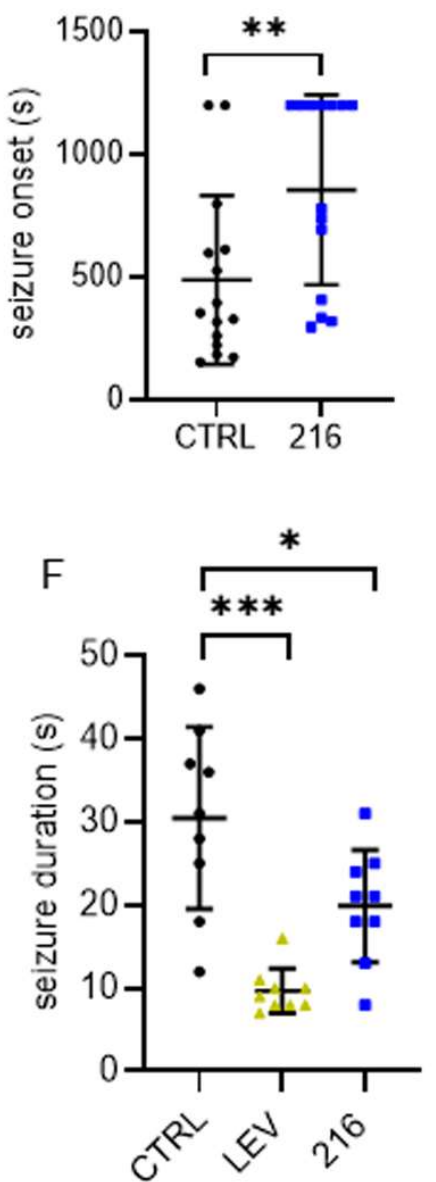

Figure 5. Characterisation of RAB216 shows it to be more potent than 4-TBB

A) Western blot analysis of the effect of exposure of mouse brain to RAB216 on Pum $1(\mathrm{n}=4, p=0.07)$ and $2(\mathrm{n}=4, p=$ $0.01)$ expression (unpaired $t$-tests). B) Sodium valproate (VPA), which is an effective anticonvulsant in this assay, had no effect on expression of Pum1 or 2 ( $\mathrm{n}=$ $4, p>0.05)$. C) Effect of RAB216 on Straub tail $(p=0.03)$ and D) first appearance of a tonic-clonic seizure $(p=$ $0.01)$ in the PTZ-induced seizure assay. Timings were capped at $20 \mathrm{~min}(1200 \mathrm{sec})$. $\mathrm{N}=15$, (unpaired $t$-tests). E) Percentage animals exposed to PTZ showing tonicclonic seizure following exposure to either saline (CTRL) or RAB216 $(p=0.05)$ respectively, $(\mathrm{n}=15$ and 14, respectively, Fishers exact test). F) Exposure to RAB2 16 reduces $6 \mathrm{~Hz}$ electrically-indued seizure duration $(\mathrm{n}=9 p=0.013)$. Levetiracetam (LEV, $100 \mathrm{mg} / \mathrm{kg}, \mathrm{n}=9, p$ $<0.0001)$ was used as a positive control with known efficacy in this assay. Significance was tested using ANOVA $\left(F_{(2,24)}=16.88\right)$ with Dunnett's post-hoc multiple comparison. $*=p<0.05, * *=p$ $<0.01, * * *=p<0.001$. 


\section{Discussion}

We report here that 4-TBB and analogues, especially RAB216, are potent anticonvulsants with a novel mode of action that involves up-regulation of the homeostatic regulator Pum. Neuronal homeostasis is likely to assume particular importance in epileptic circuits because of the extreme levels of activity associated with the condition. Notably though, homeostasis has never been specifically targeted for anticonvulsant control. An added appeal for targeting homeostatic mechanisms is that these may, realistically, be expected to impact rather less on normal physiology. As such, we predict that anticonvulsant strategies, involving neuronal homeostasis, may prove less susceptible to side effects, which are the primary reason for switching anti-epileptic medication in the clinic ${ }^{23}$. This is because homeostatic mechanisms have multiple in-built protective regulatory controls that work to prevent under- or over-activation; in the case of Pum, this protein also targets its own mRNA and at least one of its cofactors ${ }^{6}$.

Whilst multiple forms of neuronal homeostasis have been described, including synaptic scaling and presynaptic regulation of neurotransmitter release ${ }^{24,25}$, the compounds we describe seemingly manipulate firing rate homeostasis, which acts to maintain action potential firing within predetermined and physiologically relevant limits ${ }^{2}$. This is achieved, at least in part, by Pumdependent control of voltage-gated $\mathrm{Na}^{+}$channel synthesis ${ }^{1}$. Analyses of $\mathrm{Na}_{\mathrm{v}}$ protein levels, in postmortem mouse brains, pre-exposed to 4-TBB, validates this mode-of action, showing reduction in $\mathrm{Na}_{\mathrm{v}} \mathrm{S} 1.1$ and 1.2 but, interestingly, no change in $\mathrm{Na}_{\mathrm{v}}$ 1.6. Intuitively, one might predict a reduction in $\mathrm{Na}_{\mathrm{v}} 1.6$ because gain-of function mutations in the encoding gene, $\mathrm{Na}_{\mathrm{v}} 1.6$, are associated with hyperactivity and epilepsy ${ }^{26}$. By similar logic, the observed reduction of $\mathrm{Na}_{\mathrm{v}} 1.1$ is also unexpected given that this channel type predominates in GABAergic inhibitory neurons ${ }^{27}$. Our analysis of these known targets of Pum is, however, relatively crude in treating the whole brain as a single tissue. This approach similarly identifies reduced expression of the GLUR2 AMPA receptor subunit 
following exposure to 4-TBB. Again, how a reduction in this receptor subunit affects neuronal activity, particularly across the entire brain, is difficult to predict. Glutamatergic synaptic currents, in neurons with reduced expression of GLUR2, exhibit increased deactivation rates which may limit the degree of depolarization induced in the postsynaptic cell ${ }^{28}$. Whilst details remain to be resolved, the changes we observe in $\mathrm{Na}_{\mathrm{v}}$ and GLUR2 protein levels are consistent with increased Pum expression and, in this regard, serve to strengthen our hypothesis that up-regulation of this homeostatic regulator contributes to the anticonvulsant effect of 4-TBB and RAB216.

We can at present only speculate on how 4-TBB-like molecules mediate an increase in Pum expression. Indeed, in this regard, it is interesting to consider how neurons monitor their activity which, in turn, is transduced to regulate the activity status of intrinsic homeostatic mechanisms. In the case of Pum, we have reported, in Drosophila, that synaptic depolarization regulates expression of $\mathrm{p} 300$, a histone acetyltransferase that forms a complex with Mef2. As synaptic depolarization increases, levels of p300 reduce, releasing Mef2 from the complex. Once released, Mef2 binds the dpum promoter and transactivates gene transcription ${ }^{19}$. In mammals, by contrast, the level of Mef2 expression is itself activity-regulated, increasing with depolarization ${ }^{29}$, and analysis of human and mouse pum 2 promoters identifies multiple Mef2 binding motifs ${ }^{19} \cdot \mathrm{p} 300$ is also reported to regulate Mef2 in mammals ${ }^{30}$, but how this protein is influenced by synaptic depolarization has not been described. In mammals, Mef2 also increases the expression of micro-RNAs, including miR-134, which is sufficient to down-regulate expression of Pum2 ${ }^{31,32}$. Significantly, block of miR-134, using an antagomir, is anticonvulsive in rodents ${ }^{33}$. Thus, it is conceivable that 4-TBB, and analogues, might act at any level throughout this seemingly complex regulatory mechanism that ensures appropriate expression of Pum proteins. It is expected that levels of Pum are tightly regulated given the requirement to guard against under or over-activity of neuron activity. Indeed, these extensive regulatory and feedback controls, present in Pum-dependent homeostasis, may 
prove beneficial in exploiting this system for anticonvulsive therapy: minimising potential sideeffects of exposure to 4-TBB or its analogues.

In summary, the study we report here provides a first proof-of-principle that manipulation of neuronal homeostasis, and in particular Pum, provides an exploitable route to suppress seizures and, moreover, may be suitable for the treatment of patients that have drug-refractory seizures.

\section{Acknowledgements}

We thank Aoibhinn Kelly, Iona Hayes, Thomas Humphreys and Ceri Hughes who contributed to the 4-TBB structure-activity assay as part of their final year BSc. Projects. This work was supported by funding from the Biotechnology and Biological Sciences Research Council (BB/L027690/1 to RAB); Epilepsy Research UK (PGE2002, Explore Pilot Grant to RAB, RW and SA); an MRCfunded PhD studentship (to FM), a Wellcome Trust-NIH PhD studentship (205944/Z/17/Z to CMGS), the Vrije Universiteit Brussel (to NA and IS) and by MRC (MR/R005427/1 to AT). Work on this project benefited from the Manchester Fly Facility, established through funds from the University and the Wellcome Trust (087742/Z/08/Z).

\section{Author contributions}

Collected data: FM, WHL, CMGS, NA, YNF, GC. RP. Analyzed data: FM, WHL, CMGS, NA, RAB. Supervised experiments: IS, AT, RW, SA, SF, RAB. Wrote manuscript: RB, AT

\section{Disclosure}

None of the authors have any conflicts of interest to disclose.

\section{Data availability statement}

All research data supporting this publication are directly available within this publication. 


\section{Materials and correspondence:}

\section{Richard.Baines@manchester.ac.uk}

\section{Materials and methods}

Animals: Drosophila were maintained at $25^{\circ} \mathrm{C}$ on a 16:8 light/dark cycle. Mice were housed on a 12:12 light/dark cycle at a constant ambient temperature of $21 \pm 2{ }^{\circ} \mathrm{C}$ and given access to water and diet ad libitum. All procedures were conducted in accordance with local institutional policies and guidelines. All procedures undertaken at Manchester were approved by the University of Manchester Animal Welfare and Ethics Board and conducted in accordance with project licence authority granted under the Animals (Scientific Procedures) Act 1986.

Seizure behaviour test in Drosophila: Wall-climbing, third-instar larvae (L3), of either sex, were subjected to an electric shock (4V DC, 3s) to induce seizure, with or without previous feeding of compound, as described ${ }^{34}$. Recovery times (RT) are shown which depict the time taken for larvae to recover, evidenced by a full peristaltic wave and normal locomotion. A cut-off time of 420 s was used. For compound-feeding studies, eggs were laid on food containing compound (or vehicle, $0.4 \% \mathrm{DMSO}$ ) and larvae were raised (in the presence of drug) until L3. Where experiments were conducted over a number of weeks (e.g. analogue screen shown in Fig. 4), RT was normalized to the para $^{\text {bss }}$ (without compound) run each week.

dPum:promoter assay: $A$ dpum promoter-GAL4 line ${ }^{19}$ was crossed to attP24 UAS-luciferase flies

35. Flies carrying the UAS-luciferase transgene alone were used for background controls. Adult flies were allowed to lay eggs in vials containing food with added compound (or vehicle, DMSO) and to develop to L3. Ten L3 CNSs, of either sex, were placed in $100 \mu 1$ Promega Glo Lysis buffer for each sample, and 5 independent samples collected. CNSs were homogenized, incubated at room temperature $(10 \mathrm{~min})$, centrifuged $(5 \mathrm{~min})$, and supernatant transferred to a new tube. $30 \mu \mathrm{l}$ of each 
sample was then transferred to a well of a white-walled 96-well plate at room temperature, $30 \mu 1$ Promega Luciferase reagent was added to each well and plates incubated in the dark (10 min). Luminescence was measured with a GENios plate reader (TECAN, Reading, UK). Values were normalized to total protein concentration, measured using the Bradford protein assay (Bio-Rad, Watford, UK).

Quantitative RT-PCR: QRT-PCR was performed using a SYBR Green I real-time PCR method (Roche, LightCycler ${ }^{\circledR} 480$ SYBR Green I Master, Mannheim, Germany) as described ${ }^{36}$. RNA was extracted from 20 L3 CNSs per replicate, of either sex, using the RNeasy micro kit (QIAGEN, Hilden, Germany). Primer sequences (5' to 3') were: actin-5C (CG4027), CTTCTACAATGAGCTGCGT and GAGAGCACAGCCTGGAT; dpum (CG9755), GCAGCAGGGTGCCGAGAATC and CGCGGCGACCCGTCAACG (forward and reverse, respectively). Relative gene expression was calculated as the $2^{-\Delta \mathrm{Ct}}$, where $\Delta \mathrm{Ct}$ was determined by subtracting the average actin-5C Ct value from that of $d p u m$.

Organotypic slice cultures: Slice cultures were prepared from 5 to 9 day old C57BL/6J mouse pups, of either sex, according to the interface organotypic culture method ${ }^{37,38}$. Brains were removed and hippocampi dissected and transversely sectioned into $350 \mu \mathrm{m}$ slices (McIlwain tissue chopper). Slices were plated on polyester membrane inserts $(0.4 \mu \mathrm{m}$ pore) in 6 well culture plates (Corning Costar CLS3450-24EA, Sigma-Aldrich, UK), 2-3 slices per insert, containing 1.2ml of feeding media (50\% Minimum Essential Media + GlutaMAX, phenol red and with Earle's salts (Fisher Scientific, Loughborough, UK), $25 \%$ heat-inactivated horse serum (Sigma, Poole, UK), $21.56 \%$ EBSS, $2 \%$ B27 serum (Fisher Scientific) and $36 \mathrm{mM}$ D-Glucose. Slices were kept at $37^{\circ} \mathrm{C} / 5 \%$ $\mathrm{CO}_{2}$ and the media replaced the day after plating and then 2-3 times weekly depending on plating density. This method of preparing organotypic hippocampal slice cultures induces spontaneous epileptic-like activity without the need for any pharmacological or electrical provocation. The 
slicing process mimics a traumatic brain injury which leads to cell death, deafferentation and subsequent axonal sprouting - all of which contribute towards the gradual development of seizurelike activity ${ }^{39}$.

Local field potentials, at Days In Vitro (DIV) 7-14, were recorded (sampling rate $10 \mathrm{kHz}$ ) in CA1 using glass borosilicate patch pipettes ( 1-3M $\Omega$, Harvard Apparatus, Kent, UK) and a Multiclamp 700B (Molecular Devices, CA, USA). Slices were perfused with oxygenated ACSF (125 mM NaCl, $26 \mathrm{mM} \mathrm{NaHCO}_{3}, 10 \mathrm{mM}$ glucose, $3.5 \mathrm{mM} \mathrm{KCl}, 1.26 \mathrm{mM} \mathrm{NaH}_{2} \mathrm{PO}_{4}, 2 \mathrm{mM} \mathrm{CaCl}_{2}, 1 \mathrm{mM} \mathrm{MgCl}_{2}$ ) and maintained at $33-36^{\circ} \mathrm{C}$ for 7 hours. The first hour was used as an activity baseline: slices lacking seizure-like discharges were discarded. After the first hour of baseline activity, slices were bathed in media supplemented with 4-TBB (1.2 mM). All seizure-like events, greater than five seconds, were counted. Analysis was performed using MATLAB (The MathWorks Inc., Natick, MA, USA).

PTZ seizure-induction: Mice (male, C57BL/6J, 12-15 weeks, 23-30 g,) were injected subcutaneously (sc.) with $0.1 \mathrm{ml}$ of compound (in $\mathrm{NaCl}, 0.9 \% \mathrm{w} / \mathrm{v}$ saline) or saline vehicle, once per day for 3 days. Four hours after the last injection on day 3, a single dose of PTZ $(60 \mathrm{mg} / \mathrm{kg} / \mathrm{sc}$. in saline.) was injected. Each mouse was placed into a separate clear plastic arena and videoed for $20 \mathrm{~min}$. After the observation period, mice were anesthetized with isoflurane (3-4\% in $20 \% \mathrm{O}_{2}$ and $50 \% \mathrm{~N}_{2} \mathrm{O}, 0.5 \mathrm{l} / \mathrm{min}$ ), transcardially perfused with $0.9 \%$ saline and brains removed and stored at $80^{\circ} \mathrm{C}$. Seizure scoring was carried out from videos, independently scored by two experimenters blinded to the experimental groups until full analysis was complete.

6Hz seizure-induction: NMRI mice (35-45g, male, Charles River, Chatillon-sur-Chalaronne, France) were used. Prior to the electrical stimulation, $0.5 \%$ xylocaine was applied to the cornea to induce local anesthesia and ensure good conductivity. Corneal stimulation ( $46 \mathrm{~mA}, 0.2 \mathrm{~ms}$ duration pulses at $6 \mathrm{~Hz}$ for $3 \mathrm{~s}$ ) was administered by a constant current device (ECT Unit 57800; Ugo Basile, 
Comerio, Italy) ${ }^{40,41}$. Acutely evoked 6-Hz seizures were characterized by stun, forelimb clonus, twitching of vibrissae, and/or Straub-tail. For each animal, the total seizure duration was manually recorded. Ip administration of levetiracetam (LEV, 100mg/kg) $1 \mathrm{~h}$ before seizure induction was used as a positive control ${ }^{42}$. Seizure scoring was carried out live during the experiment. The entire experiment was also video recorded. The researcher was blinded to the experimental groups until full analysis was complete.

Western blot: Whole brain was homogenised in ice-cold buffer $(150 \mathrm{mM} \mathrm{NaCl}, 50 \mathrm{mM}$ Tris- $\mathrm{HCl}$, $1 \%$ Nonidet P-40, 0.5\% Sodium deoxycholate and $0.1 \%$ SDS) containing protease inhibitors (Promega, Madison, USA) and centrifuged at $10,000 \times \mathrm{g}\left(30 \mathrm{~min}\right.$ at $\left.4^{\circ} \mathrm{C}\right)$. Supernatant was stored at $-20^{\circ} \mathrm{C}$. Antibodies were: anti-Pum 1 (1:1000, \#12322, Cell Signaling, MA, USA), anti-Pum 2 (1:1000, ab10361, Abcam, Cambridge, UK), anti-SCN1A (1:1000, ASC-001, alomone labs, Jerusalem, Israel), anti-SCN2A (1:1000, ASC-002, alomone labs), anti-SCN8A (1: 1000, ASC-009, alomone labs), anti-GluR2 (1:2000, AB1768-I, Merck, Darmstadt, Germany) and anti- $\beta$-Actin (1:5000, ab8227, Abcam). Samples (25 $\mu \mathrm{g}$ of protein) were separated by SDS page, and protein transferred to a polyvinylidene difluoride membrane (GE Healthcare). After blocking (0.5\% BSA and $0.05 \%$ TWEEN-20 in Tris-buffered saline, TBS-T), membrane was incubated overnight $\left(4^{\circ} \mathrm{C}\right)$ in primary antibody diluted in $0.5 \%$ BSA in TBS-T. Membranes were incubated with HRPconjugated secondary antibodies (1:2500, \#7074, Cell Signaling) in 0.3\% BSA in TBS-T and blots developed with an Enhanced Chemiluminescent Detection Kit (Pierce, Rockford, USA). Protein band density was measured using Image J (NIH, USA).

Chemical synthesis: RAB216 was designed by first screening analogues of 4-TBB and its carboxylic acid derivative RAB102 (data not shown). The structure activity relationship (SAR) revealed that only compounds with a carboxylic acid or aldehyde group directly bonded to the benzene ring were active, even when replaced with isosteric groups. Furthermore, a change of the 
bioRxiv preprint doi: https:/doi org/10.1101/2022.03.07.483229; this version posted March 7 2022. The copyright holder for this preprint (which was not certified by peer review) is the author/funder, who has granted bioRxiv a license to display the preprint in perpetuity. It is made available under aCC-BY 4.0 International license.

benzene ring to a pyridine or indole ring, or a change of substituents from para to meta, resulted in complete loss of activity. Electronegative groups were tolerated in the para-position, increasing the likelihood of a compound forming strong intermolecular bonds with its binding partner. Therefore, when designing the second-generation compounds (sTable 1), some analogues included electronegative oxygen and nitrogen atoms. This included RAB216 which contained a pyrazole ring, providing extra interactions to enhance activity. Additionally, analysis of the screen showed that compounds with an element of 3D structure were more active: in RAB216 the two methyl groups attached to the pyrazole group caused it to be twisted by approximately $18^{\circ}$. Reviews of drug libraries suggest that planar compounds are less likely to be biologically active ${ }^{43}$. Compounds, including RAB216, were designed in accordance with Lipinski's rules, such as molecular weight (under 500) and lipophilicity (logP under 5), to give desirable physiochemical properties ${ }^{44}$. All of the compounds screened, including RAB216, fit within these guidelines (sTable 1).

Statistics: Statistical significance was tested using either a Student's two-tailed $t$-test (paired or unpaired), a one-way or two-way ANOVA followed by post-hoc testing (multiple experimental groups) or Fishers exact test. Level of significance on figures is indicated by * $(p \leq 0.05), * *(p \leq$ $0.01), * * *(p \leq 0.001)$. Figures show means $\pm \mathrm{SD}$.

\section{References}

1 Giachello, C. N. \& Baines, R. A. Regulation of motoneuron excitability and the setting of homeostatic limits. Curr Opin Neurobiol 43, 1-6, doi:10.1016/j.conb.2016.09.014 (2017).

2 Driscoll, H. E., Muraro, N. I., He, M. \& Baines, R. A. Pumilio-2 regulates translation of nav1.6 to mediate homeostasis of membrane excitability. J Neurosci 33, 9644-9654, doi:10.1523/jneurosci.0921-13.2013 (2013).

3 Mee, C. J., Pym, E. C., Moffat, K. G. \& Baines, R. A. Regulation of neuronal excitability through pumilio-dependent control of a sodium channel gene. J Neurosci 24, 8695-8703 (2004).

4 Gerber, A. P., Luschnig, S., Krasnow, M. A., Brown, P. O. \& Herschlag, D. Genome-wide identification of mRNAs associated with the translational regulator PUMILIO in Drosophila melanogaster. Proc Natl Acad Sci U S A 103, 4487-4492 (2006). 
5 Zhang, M. et al. Post-transcriptional regulation of mouse neurogenesis by Pumilio proteins. Genes Dev 31, 1354-1369, doi:10.1101/gad.298752.117 (2017).

6 Muraro, N. I. et al. Pumilio binds para mRNA and requires Nanos and Brat to regulate sodium current in Drosophila motoneurons. J Neurosci 28, 2099-2109, doi:28/9/2099 [pii] 10.1523/JNEUROSCI.5092-07.2008 (2008).

7 Arvola, R. M., Weidmann, C. A., Tanaka Hall, T. M. \& Goldstrohm, A. C. Combinatorial control of messenger RNAs by Pumilio, Nanos and Brain Tumor Proteins. RNA Biol, 1-12, doi:10.1080/15476286.2017.1306168 (2017).

8 Dong, H. et al. Pumilio2 regulates synaptic plasticity via translational repression of synaptic receptors in mice. Oncotarget 9, 32134-32148, doi:10.18632/oncotarget.24345 (2018).

9 Patsalos, P. N. The clinical pharmacology profile of the new antiepileptic drug perampanel: A novel noncompetitive AMPA receptor antagonist. Epilepsia 56, 12-27, doi:10.1111/epi.12865 (2015). Galgano, A. et al. Comparative analysis of mRNA targets for human PUF-family proteins suggests extensive interaction with the miRNA regulatory system. PLOS ONE 3, e3164, doi:10.1371/journal.pone.0003164 (2008).

11 Hogan, G. J., Brown, P. O. \& Herschlag, D. Evolutionary Conservation and Diversification of Puf RNA Binding Proteins and Their mRNA Targets. PLoS Biol 13, e1002307, doi:10.1371/journal.pbio.1002307 (2015).

12 Bohn, J. A. et al. Identification of diverse target RNAs that are functionally regulated by human Pumilio proteins. Nucleic Acids Res 46, 362-386, doi:10.1093/nar/gkx1120 (2018). Gennarino, V. A. et al. Pumilio1 haploinsufficiency leads to SCA1-like neurodegeneration by increasing wild-type Ataxin1 levels. Cell 160, 1087-1098, doi:10.1016/j.cell.2015.02.012 (2015).

14 Follwaczny, P. et al. Pumilio2-deficient mice show a predisposition for epilepsy. Dis Model Mech 10, 1333-1342, doi:10.1242/dmm.029678 (2017).

15 Siemen, H., Colas, D., Heller, H. C., Brustle, O. \& Pera, R. A. Pumilio-2 function in the mouse nervous system. PLOS ONE 6, e25932, doi:10.1371/journal.pone.0025932 (2011).

$16 \mathrm{Wu}, \mathrm{X}$. L. et al. Reduced Pumilio-2 expression in patients with temporal lobe epilepsy and in the lithium-pilocarpine induced epilepsy rat model. Epilepsy Behav 50, 31-39, doi:10.1016/j.yebeh.2015.05.017 (2015).

17 Lin, W. H., Giachello, C. N. \& Baines, R. A. Seizure control through genetic and pharmacological manipulation of Pumilio in Drosophila: a key component of neuronal homeostasis. Dis Model Mech 10, 141-150, doi:10.1242/dmm.027045 (2017).

18 Baines, R. A., Giachello, C. N. G. \& Lin, W. H. Drosophila. Models of Seizures and Epilepsy, 2nd Edition, 345-358, doi:10.1016/b978-0-12-804066-9.00024-9 (2017).

19 Lin, W. H. \& Baines, R. A. Myocyte enhancer factor-2 and p300 interact to regulate the expression of homeostatic regulator Pumilio in Drosophila. Eur J Neurosci, doi:10.1111/ejn.14357 (2019). Yaari, Y., Selzer, M. E. \& Pincus, J. H. Phenytoin: mechanisms of its anticonvulsant action. Ann Neurol 20, 171-184, doi:10.1002/ana.410200202 (1986).

21 Vessey, J. P. et al. Mammalian Pumilio 2 regulates dendrite morphogenesis and synaptic function. Proc Natl Acad Sci U S A 107, 3222-3227, doi:10.1073/pnas.0907128107 (2010).

22 Loscher, W. Critical review of current animal models of seizures and epilepsy used in the discovery and development of new antiepileptic drugs. Seizure 20, 359-368, doi:10.1016/j.seizure.2011.01.003 (2011).

23 Kwan, P., Schachter, S. C. \& Brodie, M. J. Drug-resistant epilepsy. N Engl J Med 365, 919-926, doi:10.1056/NEJMra1004418 (2011).

24 Davis, G. W. \& Bezprozvanny, I. Maintaining the stability of neural function: a homeostatic hypothesis. Annu Rev Physiol 63, 847-869, doi:10.1146/annurev.physiol.63.1.847 (2001).

25 Turrigiano, G. G. \& Nelson, S. B. Homeostatic plasticity in the developing nervous system. Nat Rev Neurosci 5, 97-107 (2004). 
bioRxiv preprint doi: https//doi.org/10.1101/2022.03.07.483229. this version posted March 7. 2022. The copvriaht holder for this preprint (which was not certified by peer review) is the author/funder, who has granted bioRxiv a license to display the preprint in perpetuity. It is made available under aCC-BY 4.0 International license.

26 O'Brien, J. E. \& Meisler, M. H. Sodium channel SCN8A (Nav1.6): properties and de novo mutations in epileptic encephalopathy and intellectual disability. Front Genet 4, 213, doi:10.3389/fgene.2013.00213 (2013).

27 Catterall, W. A., Kalume, F. \& Oakley, J. C. NaV1.1 channels and epilepsy. J Physiol 588, 1849-1859, doi:10.1113/jphysiol.2010.187484 (2010).

28 Geiger, J. R. et al. Relative abundance of subunit mRNAs determines gating and Ca2+ permeability of AMPA receptors in principal neurons and interneurons in rat CNS. Neuron 15, 193-204 (1995).

29 Flavell, S. W. et al. Activity-dependent regulation of MEF2 transcription factors suppresses excitatory synapse number. Science 311, 1008-1012, doi:10.1126/science.1122511 (2006). De Luca, A. et al. p300/cAMP-response-element-binding-protein ('CREB')-binding protein (CBP) modulates co-operation between myocyte enhancer factor 2A (MEF2A) and thyroid hormone receptor-retinoid X receptor. Biochem J 369, 477-484, doi:10.1042/bj20020057 (2003).

31 Fiore, R. et al. Mef2-mediated transcription of the miR379-410 cluster regulates activity-dependent dendritogenesis by fine-tuning Pumilio2 protein levels. Embo J 28, 697-710, doi:10.1038/emboj.2009.10 (2009).

32 Fiore, R. et al. MiR-134-dependent regulation of Pumilio-2 is necessary for homeostatic synaptic depression. Embo J 33, 2231-2246, doi:10.15252/embj.201487921 (2014).

33 Reschke, C. R. et al. Potent Anti-seizure Effects of Locked Nucleic Acid Antagomirs Targeting miR134 in Multiple Mouse and Rat Models of Epilepsy. Mol Ther Nucleic Acids 6, 45-56, doi:10.1016/j.omtn.2016.11.002 (2017).

34 Marley, R. \& Baines, R. A. Increased persistent Na+ current contributes to seizure in the slamdance bang-sensitive Drosophila mutant. J Neurophysiol 106, 18-29 (2011).

35 Markstein, M., Pitsouli, C., Villalta, C., Celniker, S. E. \& Perrimon, N. Exploiting position effects and the gypsy retrovirus insulator to engineer precisely expressed transgenes. Nat Genet 40, 476-483, doi:10.1038/ng.101 (2008).

36 Lin, W. H., He, M. \& Baines, R. A. Seizure suppression through manipulating splicing of a voltagegated sodium channel. Brain 138, 891-901, doi:10.1093/brain/awv012 (2015).

37 Gogolla, N., Galimberti, I., DePaola, V. \& Caroni, P. Preparation of organotypic hippocampal slice cultures for long-term live imaging. Nat Protoc 1, 1165-1171, doi:10.1038/nprot.2006.168 (2006).

38 Stoppini, L., Buchs, P. A. \& Muller, D. A simple method for organotypic cultures of nervous tissue. J Neurosci Methods 37, 173-182 (1991).

39 McBain, C. J., Boden, P. \& Hill, R. G. Rat hippocampal slices 'in vitro' display spontaneous epileptiform activity following long-term organotypic culture. J Neurosci Methods 27, 35-49, doi:10.1016/0165-0270(89)90051-4 (1989).

40 Aourz, N. et al. Identification of GSK-3 as a Potential Therapeutic Entry Point for Epilepsy. ACS Chem Neurosci 10, 1992-2003, doi:10.1021/acschemneuro.8b00281 (2019).

41 Walrave, L. et al. Inhibition of astroglial connexin43 hemichannels with TAT-Gap19 exerts anticonvulsant effects in rodents. Glia 66, 1788-1804, doi:10.1002/glia.23341 (2018). Walrave, L. et al. Validation of the $6 \mathrm{~Hz}$ refractory seizure mouse model for intracerebroventricularly administered compounds. Epilepsy Res 115, 67-72, doi:10.1016/j.eplepsyres.2015.06.003 (2015).

43 Lovering, F., Bikker, J. \& Humblet, C. Escape from flatland: increasing saturation as an approach to improving clinical success. J Med Chem 52, 6752-6756, doi:10.1021/jm901241e (2009).

44 Lipinski, C. A., Lombardo, F., Dominy, B. W. \& Feeney, P. J. Experimental and computational approaches to estimate solubility and permeability in drug discovery and development settings. Adv Drug Deliv Rev 46, 3-26, doi:10.1016/s0169-409x(00)00129-0 (2001). 